\title{
Research on Relaxation Theory in Piano Playing
}

\author{
Chunxiao Li
}

Henan Art Vocational College, Zhengzhou, Henan, 450053, China

\begin{abstract}
This paper analyzes the different understandings of "relaxation" in piano performance, and draws the conclusion that "natural tension" is needed in piano performance. Through effective experiments and exercises, learners can experience "natural and controlled" tension "and benefit from it.

Keywords: piano playing, relax, tension
\end{abstract}

\section{Introduction}

The piano player is probably no stranger to the word "relax," but have you ever wondered if your arm is really in a state of complete relaxation when you play the piano? The fingers can cope with the complex

Does this have to do with piano performance? Based on Seymour Bernstein's Theory of Relaxation, this article begins with an understanding of "relaxation" Through a series of experiments, this paper discusses the relationship between "relaxation" and "tension" in piano playing, and provides a new way for piano playing and teaching.

Seymour Bernstein published his book "Exploring Music with Your Hands" in 1981, which became a bestseller as soon as it was published. It has been translated into German, Russian, Japanese, Korean and other languages. The book explores "the purpose of practicing piano", "practicing piano discipline" and "performance sense of achievement."

I read Bernstein's "use your hands to explore music," a book in the process, found the book on the "relaxation" theory is quite innovative, the piano who have 
some guiding significance, so set the axillary Qiu, And joined the author's practice, written into this article, and colleagues to discuss.

\section{The misunderstanding of "relaxation theory"}

Tobias Matthay is a famous English piano educator who describes in detail the benefits of relaxation and the natural weight ("dead" weight) that is transmitted from the shoulder to the finger pad. However, he believes that even when playing the piano is a moderate sense of tension is not necessary. It is true that his exaggerated relaxation (which he even called "relaxing the Scriptures") helped a lot of pianists, since many pianists were still influenced by the strict mode of the late nineteenth and early twentieth centuries, Playing the piano is very stiff. Until now, many teachers put this good way into teaching, and loyalty to call the "Matthew way." But there are other teachers who misrepresent this way that Tobias Matthew finally made the statement: "Only a fool will fall directly on the piano keyboard!"

\section{The re - understanding of "sense of weight"}

Simo Bernstein, in "Exploring Music with Your Hands", mentions how to really simulate the weight of the arm. It was an experiment he invented when he was young: in the leather pocket into a number of about 0.75 inches (about $19 \mathrm{~mm}$ ) of the ball (also can be used to remove code), tied to his wrist, Start playing on the piano keys. Through experimentation, he can suddenly play a full and resonant tone, which he was surprised: before he played the sound is not the case. He feels the "weight" - the weight always pulls down the arm, the force of the arm forcing the finger to rest on the keyboard, and pass between the fingers - from one finger to another finger.

Simo Bernstein also let his students experience this feeling. When the students started playing on the keys, the piano sounded so that students can not help but exclaim: "I can not believe it! I played the sound even like Rubinstein playing!" Then added: "When the time will support this small experiment, because it certainly no doubt to simulate the control arm weight effect. At first, do not try to practice for more than five minutes each time. And when the muscles gradually adapt, you can then consider the amount of time to extend. Furthermore, just started practice, do not play too fast, and the volume is not too much.

\section{The dialectical unity of relaxation and tension: natural and controlled tension}

If you want to be able to control the piano playing in the arm tension, the rational use of arm weight sense, then we must first recognize the contraction of the 
muscles brought about by the feeling. You can experience this feeling in a simple way - lying on the floor, trying to reduce the body's tension. But in fact this method in practice than the description of the difficult, you may even not aware of certain muscles unwittingly doing contraction movement. The best way is to consciously guide their sense of tension, or is a tension against another tension. Try lifting the right leg, tighten the calf muscles, and then relax. By tightening the muscles, you will realize that they are in control of muscle.

If you observe the athletes to complete a specific action easily, you will find it is difficult to distinguish the process of tension and relaxation. But the athletes are far from completely relaxed when they complete their movements, and they have a complex way of controlling tension - a process that is as sophisticated as a musician can control. Through training, athletes can control the specific needs of muscle contraction. In other words, he understands that even if the effort is more than the actual need to complete the action, it is only half done, or even counterproductive.

In the piano playing, we should also pay attention to "energy reserves" problem. If you want to practice a section filled with strong chords and octaves stacked, and playing the sound to be full and resonant, and did not blow, then we should consider the issue of energy reserves. However, because you always think about how to play the sound stronger, it will make your muscles unwittingly shrink, and you even feel the body is in a state of tension. If this is the case, you simply can not achieve the desired results! The correct approach is that you should boldly relax your body to overcome the tension.

The excess tension (unintentional tension) will be accumulated as a supplement to the lack of muscle strength. And this is actually wrong. All power should be consciously organized before the hard segments come. The excess tension only has a destructive effect; organized tension is the concentration of the performance of the role of strength. In other words, the organization is definitely a useful driving factor. If you have been organized before the play has been consciously increased the tension, then you can be sure is that the completion of the work must be easy.

When the need to accurately express the emotional music, but the muscles can not naturally tension to the required level, we must consciously guide the muscle contraction. We mentioned the "joy" will lead to natural muscle contraction, not to mention the music contains all the emotions, it should automatically trigger the corresponding muscle contraction - the process is spontaneous, and natural.

\section{How to achieve "natural tension"}

\subsection{Experience the natural tension}

First of all, in order to understand the appropriate tension on the important role of playing, it is necessary to make their body experience the "natural tension." 
To adjust the body to the best efficiency of the state, we must first find the use of natural tension to the emotional music into the piano action method. You can try to use the following steps to experience:

The metronome is set to 60 , so that it coincides with your watch second hand synchronization.

Sitting in a chair, or in front of the piano, prepare four music on the side.

Right hand palm up, with his left hand picked up a sheet music, put it on the right hand palm.

In accordance with every eight seconds in the right hand to increase the order of a musical score until the four music is placed in the palm of your hand.

Now turn this process back, taking the order of one book from the hand every eight seconds until the next sheet of music is supported on the palm of your right hand.

It will repeat the above process. And this time to pay attention to increase, reduce the score, seriously experience the right hand and right

This tension should be consistent with the weight of the score.

This simple exercise allows us to experience the degree of contraction of the muscles in different degrees of intensity on the piano. Two sheet music is equivalent to playing the "weak" efforts; three of the music is equivalent to "strong" strength; four of the four music score is the same as playing the " This music is equivalent to "strong" efforts. Now if you want to apply this experiment to the piano, you need to find their own music in the familiar level of the same music levels, and the intensity level of ups and downs of the music section, and then were practicing.

The music spectrum of the experiment felt the sense of tension and relaxation. The stronger you play, the tighter your muscles are; the softer you play, the less tension your muscles need. But realize that, even if only holding music, but also the need for the corresponding muscle tension. In the need to play "weak" efforts still need a certain degree of solidity and stability. Through practice, you can make your muscles naturally do this. In other words, you can control your body, with the intensity of the ups and downs of the music and make rapid response.

\subsection{How much tension}

We know that doing any thing in life, whether it is playing the piano, or the trunk to move home from the store, require a certain degree of muscle tension. We have already discussed that it is pointless to relax when there is a need for tension; this will only result in uncontrollable muscle tension that occurs when there is a real need for tension force exceeds the weight of the box. In other words, you can not accurately estimate the degree of muscle tension needed to do one thing. In short, lifting up a grocery box requires some effort.

Excessive emotional attitudes can also lead to false tensions. For example, if you want to play loud and passionate fragments, you may be emotional and lose control of the physiological response. In other words, the strong feelings in the music dazzled the mind and your piano will become stiff, and finally even 
become "smashing" piano. So you need to play with enough strength to make the piano rough enough speed to strike the strings.

\subsection{How to overcome the playing fatigue}

"My arm is about to break!" This argument actually refers to the forearm because of tension and fatigue caused by fingers can not move. This muscle fatigue is actually caused by the muscle unconscious contraction, which is like when the leg cramp feeling, completely free from their own control of the feeling. Preventive measures to prevent arm cramps: (1) require your finger - not your arm - to assume the primary responsibility for playing during the playing process; (2) use "natural Of the tension "(see below), so that the fingers maintain a certain tension; (3) gradually make this process with endurance.

\subsection{The natural tension of the fingers}

In the need to deal with some difficult fragments, the arm and the fingers will be between the outbreaks of a "battle." Your fingers should not assume full responsibility, and your muscles will unconsciously shrink as a supplement to your fingers. Over time, your arm may cramp, and if you keep your fingers "natural tension" can make you avoid arm cramps.

The requirement to tighten your fingers is not to stiffen your fingers. Tight fingers are also flexible, while flexibility is the opposite of rigid. For example, observe the condition of your legs when standing: Although your legs are strained when you stand (when you are completely relaxed), they are mobile and therefore not stiff. It is this flexibility that allows you to control walking when walking.

\section{Conclusion}

Through the above analysis and experiments can be seen in the piano, our fingers and arms are not completely relaxed and need for a natural state of tension. This paper analyzes how to balance the relationship between tension and relaxation, how to control the "natural tension", how to guide the flow of energy in the body, and the proper allocation and release of energy according to the needs of music, hoping provide some experience for our piano playing and teaching.

\section{References}

[1] Scymour Bcrnstcin, with your own two hands-Discovery Though Music, New York, pp.68-70, 1981

[2] Tobias Matthay, The First Principles of Pianoforte Playing, pp.87- 91, 1905

[3] Tobias Matthay, Relaxation Studies, pp. 18- 20, 1908 